\title{
Herschel-ATLAS: The dust energy balance in the edge-on spiral galaxy UGC $4754^{\star}$
}

\author{
M. Baes ${ }^{1}$, J. Fritz ${ }^{1}$, D. A. Gadotti ${ }^{2}$, D. J. B. Smith ${ }^{3}$, L. Dunne ${ }^{3}$, E. da Cunha ${ }^{4}$, A. Amblard ${ }^{5}$, R. Auld ${ }^{6}$, G. J. Bendo ${ }^{7}$,
} D. Bonfield ${ }^{8}$, D. Burgarella ${ }^{9}$, S. Buttiglione ${ }^{10}$, A. Cava ${ }^{11,12}$, D. Clements ${ }^{7}$, A. Cooray ${ }^{5}$, A. Dariush ${ }^{6}$, G. de Zotti ${ }^{10,13}$, S. Dye ${ }^{6}$, S. Eales ${ }^{6}$, D. Frayer ${ }^{14}$, J. Gonzalez-Nuevo ${ }^{13}$, D. Herranz ${ }^{15}$, E. Ibar ${ }^{16}$, R. Ivison ${ }^{16}$, G. Lagache ${ }^{17,18}$, L. Leeuw ${ }^{19}$, M. Lopez-Caniego ${ }^{15}$, M. Jarvis ${ }^{8}$, S. Maddox ${ }^{3}$, M. Negrello ${ }^{20}$, M. Michałowski ${ }^{21}$, E. Pascale ${ }^{6}$, M. Pohlen ${ }^{6}$, E. Rigby ${ }^{3}$, G. Rodighiero ${ }^{22}$, S. Samui ${ }^{13}$, S. Serjeant ${ }^{20}$, P. Temi $^{19}$, M. Thompson ${ }^{8}$, P. van der Werf ${ }^{23}$,

A. Verma ${ }^{24}$, and C. Vlahakis ${ }^{23}$

(Affiliations are available in the online edition)

Received 31 March 2010 / Accepted 7 April 2010

\begin{abstract}
We use Herschel PACS and SPIRE observations of the edge-on spiral galaxy UGC 4754, taken as part of the H-ATLAS SDP observations, to investigate the dust energy balance in this galaxy. We build detailed SKIRT radiative models based on SDSS and UKIDSS maps and use these models to predict the far-infrared emission. We find that our radiative transfer model underestimates the observed FIR emission by a factor of two to three. Similar discrepancies have been found for other edge-on spiral galaxies based on IRAS, ISO, and SCUBA data. Thanks to the good sampling of the SED at FIR wavelengths, we can rule out an underestimation of the FIR emissivity as the cause for this discrepancy. Instead we support highly obscured star formation that contributes little to the optical extinction as a more probable explanation.
\end{abstract}

Key words. radiative transfer - dust, extinction - galaxies: ISM - infrared: galaxies

\section{Introduction}

Edge-on spiral galaxies are an important class of galaxies in which the distribution and properties of interstellar dust grains can be studied in great detail. The dust in these systems shows prominently as dust lanes in optical images; for several edgeon spiral galaxies, the dust distribution has been modelled by fitting realistic radiative transfer models to such optical images (Kylafis \& Bahcall 1987; Xilouris et al. 1997, 1998, 1999; Alton et al. 2004; Bianchi 2007). The conclusion of these works is that, in general, the dust disc is thinner (vertically) but radially more extended than the stellar disc and that the central optical depth perpendicular to the disc is less than one in optical wavebands, making the disc almost transparent when seen face-on.

A complementary way of studying the dust component in galaxies is through its thermal emission at far-infrared (FIR) and submm wavelengths. A self-consistent treatment of extinction and thermal emission, i.e. a study of the dust energy balance, gives the strongest constraints on the dust content of spiral galaxies. When we quantitatively compare the results from extinction studies and FIR/submm emission studies, a discrepancy is found: the relatively optically thin dust discs emerging from the optical modelling absorb about $10 \%$ of the stellar radiation, whereas FIR studies of normal spiral galaxies indicate they typically reprocess about $30 \%$ of the UV/optical radiation (Popescu $\&$ Tuffs 2002). This problem is clearly illustrated when applied to individual edge-on spiral galaxies: the predicted FIR fluxes of self-consistent radiative transfer models that successfully ex-

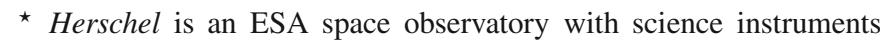
provided by European-led principal- investigator consortia and with important participation from NASA. plain the optical extinction generally underestimate the observed FIR fluxes by a factor of about three (Popescu et al. 2000; Misiriotis et al. 2001; Alton et al. 2004; Dasyra et al. 2005). Several scenarios (see Sect. 4) have been proposed to explain this discrepancy, but a major problem distinguishing between these is that the number of edge-on galaxies for which such detailed studies have been done so far is limited, owing to the poor sensitivity, spatial resolution, and limited wavelength coverage of the available FIR instruments. Herschel (Pilbratt et al. 2010) offers the possibility of studying the dust energy-balance in spiral galaxies in more detail than before. The combination of the sensitivity and the wavelength coverage of the PACS (Poglitsch et al. 2010) and SPIRE (Griffin et al. 2010) instruments, together covering the 70 to $500 \mu \mathrm{m}$ region where the emission from cold dust dominates, enables us to make reliable estimates of the total thermal emission of the interstellar dust.

A modest number of large, nearby edge-on spiral galaxies are targetted by Herschel key programs, including the Very Nearby Galaxies program and the Herschel Reference Survey (Boselli et al. 2010). Much promise, however, is offered by the Herschel Astrophysical TeraHertz Large Area Survey (H-ATLAS, Eales et al. 2010), which will image a total area of $550 \mathrm{deg}^{2}$ with PACS and SPIRE at 100, 160, 250, 350, and $500 \mu \mathrm{m}$. Based on the Revised Flat Galaxy Catalogue (Karachentsev et al. 1999) and the 2MASS-selected Flat Galaxy Catalogue (Mitronova et al. 2004), we expect between 100 and 200 resolved edge-on spiral galaxies in the H-ATLAS survey area.

In this letter, we investigate the dust energy balance in the nearby spiral galaxy UGC 4754 as a first case. This Sbc galaxy is the nearest $\left(v_{r}=5662 \pm 45 \mathrm{~km} \mathrm{~s}^{-1}\right.$; Jones et al. 2009) and largest edge-on spiral galaxy in the $16 \mathrm{deg}^{2}$ field observed during 

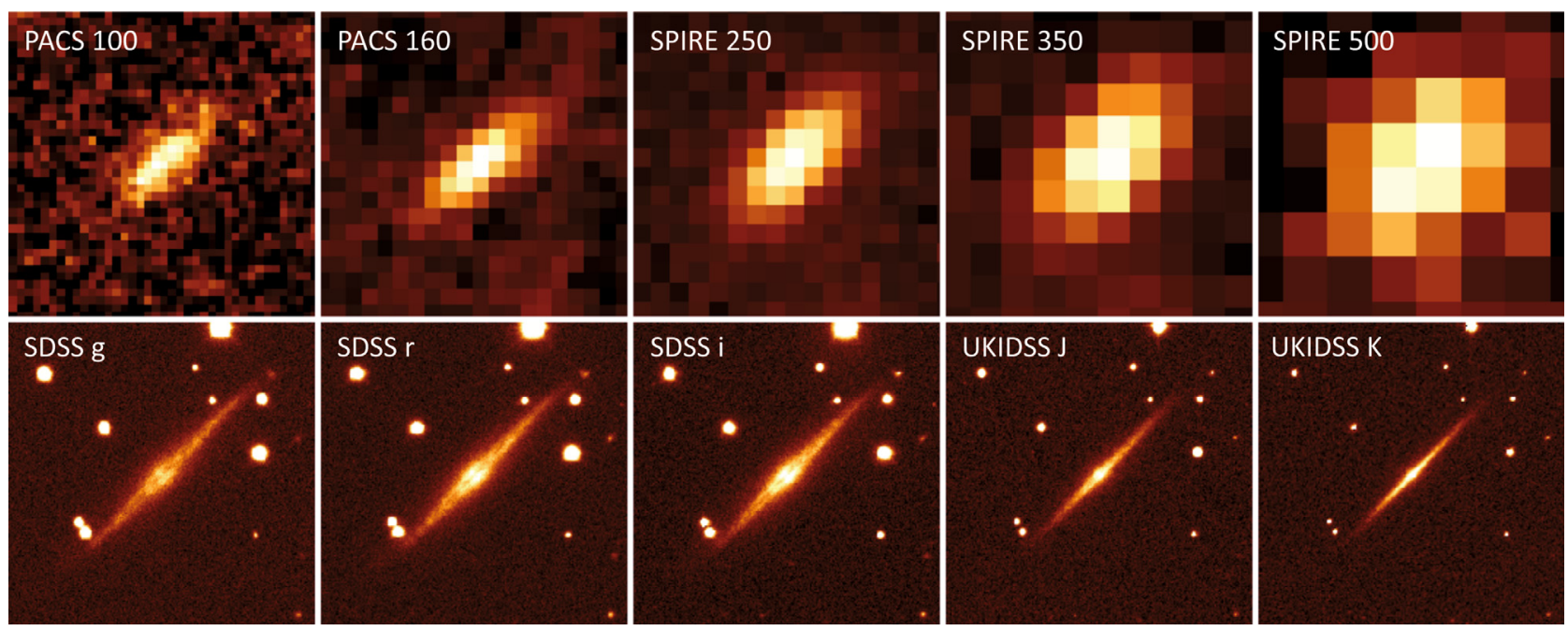

Fig. 1. The multi-wavelength view of UGC 4754. The top row shows the Herschel images, the bottom row a selection of optical and NIR images from SDSS and UKIDSS. The field-of-view of each image is $100^{\prime \prime} \times 100^{\prime \prime}$.

the H-ATLAS science demonstration phase (SDP). We present detailed radiative transfer simulations of this galaxy that fit optical and near-infrared imaging data, compute synthetic FIR fluxes and images, and compare the model predictions to the Herschel observations at 100, 160, 250, 350, and $500 \mu \mathrm{m}$. In Sect. 2 we present the observations and data reduction, in Sect. 3 we present our radiative transfer modelling and compare the model results to the observations, and in Sect. 4 we discuss these results and present out conclusions.

\section{Observations and data reduction}

The edge-on spiral galaxy UGC 4754 was observed on 2009 November 22 with the PACS and SPIRE instruments onboard Herschel as part of the H-ATLAS SDP observations of a $4 \times 4 \mathrm{deg}^{2}$ equatorial field centred on $(\alpha, \delta) \approx\left(9^{\mathrm{h}} 05^{\mathrm{m}},+0^{\circ} 30^{\prime}\right)$. Data were gathered simultaneously in the green and red PACS bands (100 and $160 \mu \mathrm{m})$ and the three SPIRE bands (250, 350 , and $500 \mu \mathrm{m})$. The PACS and SPIRE time-line data were reduced using HIPE, with reduction scripts based on the standard reduction pipelines. Maps from the SPIRE data were produced using a naive mapping technique after removing the effects of temperature variation on the timelines using a method developed by the H-ATLAS consortium (Pascale et al. 2010). Noise maps were generated by using the two cross-scan measurements to estimate the noise per detector pass, and then for each pixel the noise is scaled by the square-root of the number of detector passes. Maps from the PACS data were produced using photproject (Ibar et al. 2010).

UGC 4754 was clearly detected and spatially resolved in all the observed bands (Rigby et al. in prep.; Smith et al. in prep.). The total fluxes are $894 \pm 182 \mathrm{mJy}$ at $100 \mu \mathrm{m}, 1287 \pm 209 \mathrm{mJy}$ at $160 \mu \mathrm{m}, 878 \pm 134 \mathrm{mJy}$ at $250 \mu \mathrm{m}, 383 \pm 61 \mathrm{mJy}$ at $350 \mu \mathrm{m}$, and $122 \pm 20 \mathrm{mJy}$ at $500 \mu \mathrm{m}$. The quoted errors include statistical errors, confusion noise, and a $15 \%$ absolute calibration uncertainty (Poglitsch et al. 2010; Griffin et al. 2010).

Apart from the Herschel images, we used optical ugriz images from SDSS DR7 (Abazajian et al. 2009) and near-infrared YJHK images from UKIDSS DR5 (Lawrence et al. 2007) for our analysis. These images were obtained from the SDSS and UKIDSS archives and reduced and flux-calibrated using standard reduction methods in MIDAS. Total fluxes were measured from integrating over the flux-calibrated maps after removal of the foreground stars. Finally, we also used IRAS fluxes at 60 and $100 \mu \mathrm{m}$ from the Faint Source Catalog (Moshir et al. 1990).

\section{Analysis}

The first step in our analysis was to construct a radiative transfer model for UGC 4574, based on the gri and YJHK images; the SDSS $u$ and $z$ bands were not used in our fitting procedure because of low signal-to-noise. We used the 3D Monte Carlo radiative transfer code SKIRT for the modelling. This code was initially developed to investigate the effects of dust extinction on the photometry and kinematics of galaxies (Baes \& Dejonghe 2002; Baes et al. 2003), but has evolved to a flexible radiative transfer tool that can model the absorption, scattering, and thermal emission of circumstellar discs and dusty galaxies (e.g. Baes et al. 2005; Gadotti et al. 2010).

The stellar distribution was represented as a combination of a double exponential disc (i.e. a disc which follows an exponential profile both radially and vertically) and a flattened Sérsic model. The intrinsic stellar SED of the galaxy ${ }^{1}$ was determined by fitting a stellar population synthesis model to the dereddened SDSS-UKIDSS flux densities and adapting the code used in Hatziminaoglou et al. (2008, 2009); the resulting model SED corresponds to a population of $8 \mathrm{Gyr}$ old with an exponentially decaying star formation rate and an initial burst duration of 0.15 Gyr. The dust component was also represented as a double exponential disc. The optical properties of the dust are based on the BARE_GR_S model of Zubko et al. (2004), which accurately reproduces the extinction, emission, and abundances in the Milky Way. Our final model contains 11 free parameters: the stellar scalelength, stellar scaleheight, bulge effective radius, Sérsic index, bulge flattening, bulge-to-disc ratio, total luminosity, dust scalelength, dust scaleheight, total dust mass (or equivalently, optical depth), and inclination.

For every choice of these 11 parameters, a set of synthetic images can be constructed by solving the radiative transfer

1 To limit the numbers of parameters, we assumed a single intrinsic SED for the entire galaxy, i.e. we assumed no intrinsic colour gradients and the same SED for the bulge and disc. This assumption has virtually no effect on the predicted FIR emission, which is the main goal of this letter. 


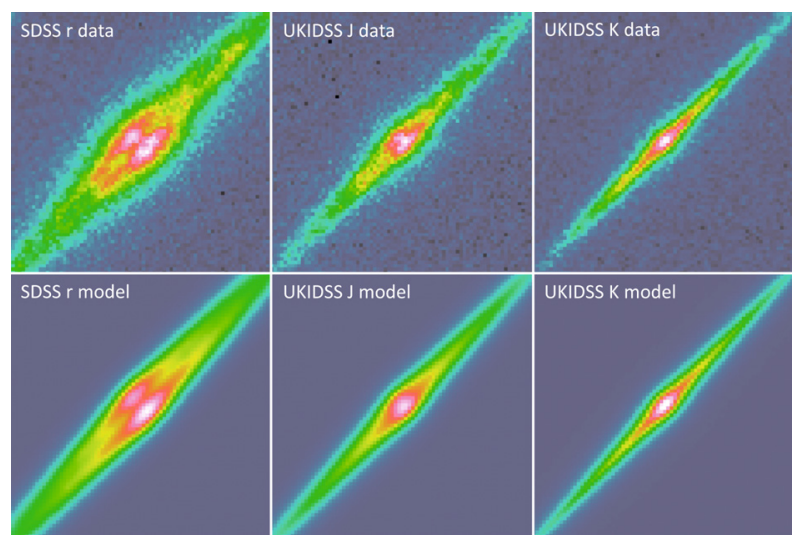

Fig. 2. Comparison of the observed $r, J$ and $K$ band images of UGC 4754 (top row) and the results of our SKIRT radiative transfer modelling (bottom row). All images show only the central $30^{\prime \prime} \times 30^{\prime \prime}$.

equation (taking both absorption and scattering into account), convolving the resulting radiation field with the appropriate transmission curves and PSF. Using a $\chi^{2}$ minimization, we looked for the set of parameters for which the corresponding synthetic images reproduced the observed images best. The initial input values for the parameters of the stellar distribution were determined from a bulge-disc decomposition of the $i$-band image using the BUDDA code (de Souza et al. 2004; Gadotti 2008). For the dust distribution, the initial input values were calculated using the average dust-star geometry from Xilouris et al. (1999). In general, our fitting approach is very similar to the method applied by Xilouris et al. (1999) and Bianchi (2007). The main difference is that we fit a single photometric model to all optical and NIR images simultaneously, whereas Xilouris et al. (1999) and Bianchi (2007) fit a new model to each different band.

Figure 2 shows the final radiative transfer model compared to the observed images in the $r, J$, and $K$ bands. The stellar disc in this model has a scalelength of $4.05 \mathrm{kpc}$ and a scaleheight of $330 \mathrm{pc}$, whereas the dust has a scalelength of $6.1 \mathrm{kpc}$ and a scaleheight of $270 \mathrm{pc}$. The total bolometric luminosity of the galaxy is $1.8 \times 10^{10} L_{\odot}$, of which the bulge only contributes $8 \%$. The total dust mass in our best-fitting model is $1.0 \times 10^{7} M_{\odot}$ and this translates into face-on optical depths (measured along the entire $z$-axis) of $0.73,0.49,0.17$ and 0.07 in the $g, r, J$, and $K$ bands, respectively. The corresponding edge-on optical depths (measured along the entire line-of-sight through the centre) are $16.5,11.1$, 3.8, and 1.6. In our model, $2.1 \times 10^{9} L_{\odot}$ or $12 \%$ of the bolometric stellar radiation is absorbed by the dust. Both the relative star-dust geometry, with a narrow and extended dust distribution, and the modest values of the optical depth agree with similar radiative transfer modelling results of other edge-on spiral galaxies (Xilouris et al. 1999; Alton et al. 2004; Bianchi 2007).

The next step in our analysis is to compare the resulting FIR emission of our radiative transfer model to the Herschel observations. Calculation of the FIR emission of a radiative transfer model is carried out using SKIRT. At each position in the galaxy, the mean intensity of the radiation field is calculated at every wavelength during the radiative transfer simulation. From this mean intensity, the equilibrium dust temperature of each species of dust grains can be calculated using the energy balance equation. We considered 10 different size bins for each of the three dust materials in the Zubko et al. (2004) model (graphite, silicate, and PAHs). We did not consider transient heating by very small grains and PAHs in our simulation, but to estimate the impact of transiently heated grains on the
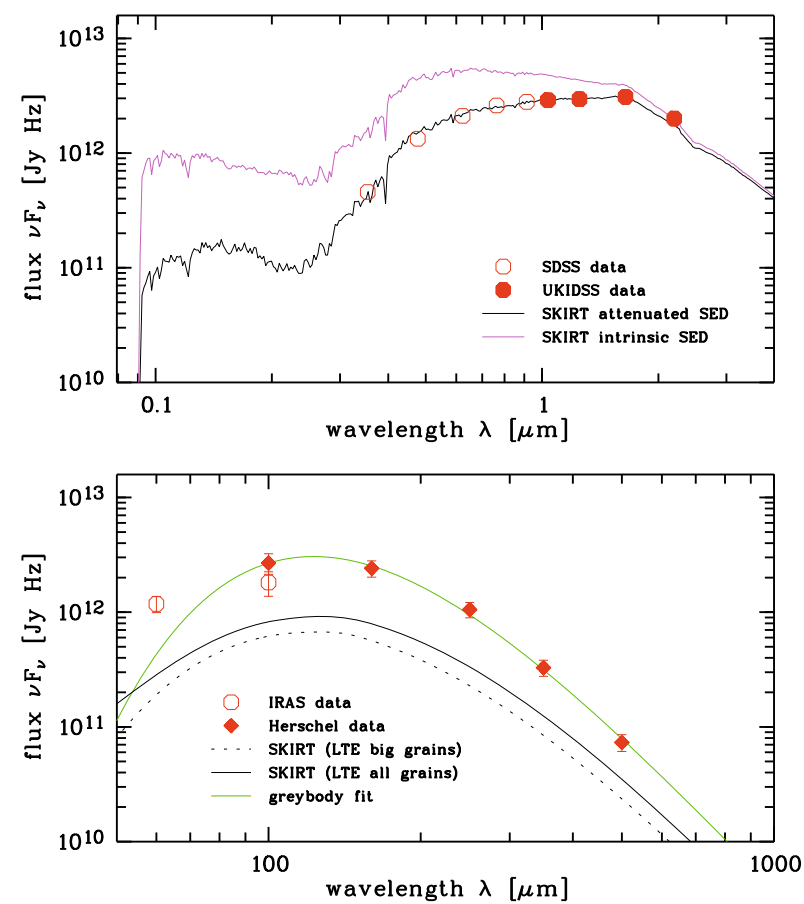

Fig. 3. The optical/NIR (top) and FIR/submm (bottom) spectral energy distribution of UGC 4754. See label and text for the meaning of the different lines.

SED in the Herschel region, we calculated the FIR emission in two ways. First, we assumed that all grains are in LTE and we added all of their thermal emission to calculate the FIR emission. Consequently, we repeated this, but only took the emission of big grains $(a>0.01 \mu \mathrm{m})$ into account.

Figure 3 shows the spectral energy distribution (SED) of UGC 4754 at optical/NIR (top panel) and FIR/submm (bottom panel) wavelengths. The solid black line in the top panel corresponds to the attenuated SED of the SKIRT model fitted to the SDSS and UKIDSS images, and the magenta line is the unattenuated SED. Our radiative transfer model reproduces the optical/NIR SED very well. In the bottom panel, the solid black line corresponds to the FIR emission of the model assuming LTE for all grains, and the dotted line represents the contribution of only the large grains. Our model significantly underestimates the observed IRAS and Herschel fluxes. This behaviour, a dust energybalance problem, has been noted for other spiral galaxies where the FIR emission calculated from radiative transfer modelling is compared to the observed IRAS, ISO, and/or SCUBA fluxes (e.g. Bianchi et al. 2000; Popescu et al. 2000; Misiriotis et al. 2001).

\section{Discussion and conclusion}

An apparently straightforward explanation to the dust energybalance problem might seem that the standard FIR emissivity, derived from dust models finetuned to the diffuse dust emission in the Milky Way, is actually underestimated by a factor two to three. This idea was advocated by Alton et al. (2000, 2004) and Dasyra et al. (2005) to explain the excess at SCUBA wavelengths of several edge-on spiral galaxies. Support for this explanation is the wide range of empirical values for the FIR emissivity that have appeared in the literature (see e.g. Hughes et al. 1997; Alton et al. 2004). Thanks to Herschel's wavelength coverage between 100 and $500 \mu \mathrm{m}$, we can rule out this possibility, because we can accurately compare the absorbed stellar 
luminosity in our radiative transfer model $\left(L_{\mathrm{abs}}=0.12 L_{\mathrm{bol}}\right)$ to the observed luminosity $L_{\mathrm{d}}$ emitted by the dust. We calculated the observed dust luminosity $L_{\text {FIR }}$ in the FIR (between $80 \mu \mathrm{m}$ and $1 \mathrm{~mm}$ ) by integrating the SED of the best-fitting modified blackbody model to the Herschel data. This model, indicated as the green line in the bottom panel of Fig. 3, corresponds to a modified blackbody model with $M_{\mathrm{d}}=1.86 \times 10^{7} M_{\odot}$ and $T=19.6 \mathrm{~K}$ and yields $L_{\mathrm{FIR}}=5.0 \times 10^{9} L_{\odot}=0.27 L_{\mathrm{bol}}$. Note that $L_{\mathrm{FIR}}$ is a lower limit to $L_{\mathrm{d}}$, since the mid-infrared luminosity (dominated by non-equilibrium emission by very small grains and PAHS) has not yet been taken into account. These numbers clearly indicate that there is a real discrepancy in the energy balance. This discrepancy cannot be lifted by changing the FIR emissivity at some wavelength; indeed, the energy balance equation impies that the ratio $L_{\mathrm{abs}} / L_{\mathrm{d}}$ is independent of the normalization of the emissivity.

The most likely explanation for this energy balance problem is that a sizable fraction of the FIR/submm emission arises from additional dust that has a negligible extinction on the bulk of the starlight. One option is the presence of a second, thinner dust disc, originally proposed by Popescu et al. (2000). Such a configuration has been successful in explaining the energy balance of spiral galaxies (Misiriotis et al. 2001; Popescu et al., in prep.) and the observed attenuation-inclination relation (Driver et al. 2007), but its validity has been questioned based on deep $K$ band images of NGC 891 (Dasyra et al. 2005). An alternative hidden dust source are young stars deeply embedded in dusty molecular clouds. The compact dust clumps can boost the FIR/submm emission of the dust, while keeping the extinction relatively unaltered (e.g. Silva et al. 1998; Bianchi et al. 2000; Popescu \& Tuffs 2005; Bianchi 2008; Michałowski et al. 2008).

A first indication that embedded star-forming clouds might be the solution to the case of UGC 4574 is that the discrepancy between our radiative transfer model and the observed FIR SED is stronger at shorter than at longer wavelengths. This implies that warmer dust (such as in star-forming regions) is necessary to bring the model in balance with the data. Further evidence is the forthcoming study by Smith et al. (in prep.) who analyse the SED of 1260 galaxies from the H-ATLAS SDP field using the technique of da Cunha et al. (2008). This technique consists of fitting the observed SED with a combination of attenuated stellar emission, dust emission from star forming-regions, and dust emission from the general ISM. For UGC 4754, Smith et al. (in prep.) find that more than $50 \%$ of the bolometric luminosity of the galaxy is reprocessed by dust. In particular, their modelling predicts a significantly bluer intrinsic UV continuum. The very efficient absorption of this radiation in star-forming regions can power the FIR luminosity, while almost remaining unnoticed in large-scale optical extinction maps. Combining the results from that study with ours, we conclude that embedded star formation is the most likely way to lift the energy balance in UGC 4754.

The present case study of UGC 4754 is a demonstration of what we can learn from detailed dust energy balance studies of edge-on spiral galaxies. In future contributions we will apply similar modelling to larger sets of edge-on galaxies and investigate possible systematic links with other galaxy parameters; for example, one would expect a correlation between the strength of the dust energy balance problem and the star formation rate if embedded star formation is the main driver (e.g. da Cunha et al. 2008, 2010). The Herschel observatory and, particularly, the H-ATLAS project offer the possibility to perform such studies in a systematic way.

Acknowledgements. This work used data from the UKIDSS DR5 and SDSS DR7. The UKIDSS project is defined in Lawrence et al. (2007) and uses the UKIRT Wide Field Camera (WFCAM; Casali et al. 2007). Funding for the SDSS and SDSS-II has been provided by the Alfred P. Sloan Foundation, the Participating Institutions, the National Science Foundation, the US Department of Energy, the National Aeronautics and Space Administration, the Japanese Monbukagakusho, the Max Planck Society, and the Higher Education Funding Council for England.

\section{References}

Abazajian, K. N., Adelman-McCarthy, J. K., Agüeros, M. A., et al. 2009, ApJS, 182,543

Alton, P. B., Xilouris, E. M., Bianchi, S., Davies, J., \& Kylafis, N. 2000, A\&A, 356, 795

Alton, P. B., Xilouris, E. M., Misiriotis, A., Dasyra, K. M., \& Dumke, M. 2004, A\&A, 425, 109

Baes, M., \& Dejonghe, H. 2002, MNRAS, 335, 441

Baes, M., Davies, J. I., Dejonghe, H., et al. 2003, MNRAS, 343, 1081

Baes, M., Dejonghe, H., \& Davies, J. I. 2005, The Spectral Energy Distributions of Gas-Rich Galaxies: Confronting Models with Data, 761, 27

Bianchi, S. 2007, A\&A, 471, 765

Bianchi, S. 2008, A\&A, 490, 461

Bianchi, S., Davies, J. I., \& Alton, P. B. 2000, A\&A, 359, 65

Boselli, A., Eales, S., Cortese, L., et al. 2010, PASP, 122, 261

Casali, M., Adamson, A., Alves de Oliveira, C., et al. 2007, A\&A, 467, 777

da Cunha, E., Charlot, S., \& Elbaz, D. 2008, MNRAS, 388, 1595

da Cunha, E., Eminian, C., Charlot, S., \& Blaizot, J. 2010, MNRAS, 403, 1894

Dasyra, K. M., Xilouris, E. M., Misiriotis, A., \& Kylafis, N. D. 2005, A\&A, 437, 447

Desert, F.-X., Boulanger, F., \& Puget, J. L. 1990, A\&A, 237, 215

de Souza, R. E., Gadotti, D. A., \& dos Anjos, S. 2004, ApJS, 153, 411

Driver, S. P., Popescu, C. C., Tuffs, R. J., et al. 2007, MNRAS, 379, 1022

Eales, S., Dunne, L., Clements, D., et al. 2010, PASP, 122, 499

Gadotti, D. A. 2008, MNRAS, 384, 420

Gadotti, D. A., Baes, M., \& Falony, S. 2010, MNRAS, 403, 2053

Griffin, M. J., et al. 2010, A\&A, 518, L3

Hatziminaoglou, E., Fritz, J., Franceschini, A., et al. 2008, MNRAS, 386, 1252

Hatziminaoglou, E., Fritz, J., \& Jarrett, T. H. 2009, MNRAS, 399, 1206

Hughes, D. H., Dunlop, J. S., \& Rawlings, S. 1997, MNRAS, 289, 766

Ibar, E., Ivison, R. J., Cava, A., et al. 2010, MNRAS, submitted

Jones, D. H., Read, M. A., Saunders, W., et al. 2009, MNRAS, 399, 683

Karachentsev, I. D., Karachentseva, V. E., Kudrya, Yu. N., Sharina, M. E., \& Parnovskij, S. L. 1999, Bull. Special Astrophys. Obs., 47, 5

Kylafis, N. D., \& Bahcall, J. N. 1987, ApJ, 317, 637

Lawrence, A., Warren, S. J., Almaini, O., et al. 2007, MNRAS, 379, 1599

Michałowski, M. J., Hjorth, J., Castro Cerón, J. M., \& Watson, D. 2008, ApJ,

672,817

Misiriotis, A., Popescu, C. C., Tuffs, R., \& Kylafis, N. D. 2001, A\&A, 372, 775

Mitronova, S. N., Karachentsev, I. D., Karachentseva, V. E., Jarrett, T. H., \&

Kudrya, Yu. N. 2004, Bull. Special Astrophys. Obs., 57, 5

Moshir, M., et al. 1990, IRAS Faint Source Catalogue, version 2.0, 0

Pascale, E., Auld, R., Dariush, A., et al. 2010, MNRAS, submitted

Pilbratt, G. L., et al. 2010, A\&A, 518, L1

Poglitsch, A., et al. 2010, A\&A, 518, L2

Popescu, C. C., \& Tuffs, R. J. 2002, MNRAS, 335, L41

Popescu, C. C., \& Tuffs, R. J. 2005, The Spectral Energy Distributions of Gas-Rich Galaxies: Confronting Models with Data, 761, 155

Popescu, C. C., Misiriotis, A., Kylafis, N. D., Tuffs, R. J., \& Fischera, J. 2000, A\&A, 362, 138

Silva, L., Granato, G. L., Bressan, A., \& Danese, L. 1998, ApJ, 509, 103

Xilouris, E. M., Kylafis, N. D., Papamastorakis, J., Paleologou, E. V., \& Haerendel, G. 1997, A\&A, 325, 135

Xilouris, E. M., Alton, P. B., Davies, J. I., et al. 1998, A\&A, 331, 894

Xilouris, E. M., Byun, Y. I., Kylafis, N. D., Paleologou, E. V., \& Papamastorakis, J. 1999, A\&A, 344, 868

Zubko, V., Dwek, E., \& Arendt, R. G. 2004, ApJS, 152, 211

Page 5 is available in the electronic edition of the journal at http://wWw . aanda. org 
1 Sterrenkundig Observatorium, Universiteit Gent, Krijgslaan 281 S9, 9000 Gent, Belgium

e-mail: maarten.baes@ugent.be

2 European Southern Observatory, Alonso de Cordova 3107, Vitacura, Santiago, Chile

3 School of Physics and Astronomy, University of Nottingham, University Park, Nottingham NG7 2RD, UK

${ }^{4}$ Department of Physics, University of Crete, PO Box 2208, 71003 Heraklion, Greece

5 Dept. of Physics \& Astronomy, University of California, Irvine, CA 92697, USA

6 School of Physics and Astronomy, Cardiff University, The Parade, Cardiff, CF24 3AA, UK

7 Astrophysics Group, Imperial College, Blackett Laboratory, Prince Consort Road, London SW7 2AZ, UK

${ }^{8}$ Centre for Astrophysics Research, Science and Technology Research Centre, University of Hertfordshire, Herts AL10 9AB, UK

${ }^{9}$ Laboratoire d'Astrophysique de Marseille, UMR6110 CNRS, 38 Rue F. Joliot-Curie, 13388 Marseille, France

10 INAF - Osservatorio Astronomico di Padova, Vicolo Osservatorio 5, 35122 Padova, Italy

11 Instituto de Astrofísica de Canarias, C/Vía Láctea s/n, 38200 La Laguna, Tenerife, Spain

12 Departamento de Astrofísica, Universidad de La Laguna (ULL), 38205 La Laguna, Tenerife, Spain
13 Scuola Internazionale Superiore di Studi Avanzati, via Beirut 2-4, 34151 Triest, Italy

14 Infrared Processing and Analysis Center, California Institute of Technology, 770 South Wilson Av, Pasadena, CA 91125, USA

15 Instituto de Física de Cantabria (CSIC-UC), Santander, 39005, Spain

16 UK Astronomy Technology Center, Royal Observatory Edinburgh, Edinburgh, EH9 3HJ, UK

17 Institut d'Astrophysique Spatiale (IAS), Bâtiment 121, 91405 Orsay, France

18 Université Paris-Sud 11 and CNRS (UMR 8617), France

19 Astrophysics Branch, NASA Ames Research Center, Mail Stop 245-6, Moffett Field, CA 94035, USA

20 Dept. of Physics and Astronomy, The Open University, Milton Keynes, MK7 6AA, UK

21 Scottish Universities Physics Alliance, Institute for Astronomy, University of Edinburgh, Royal Observatory, Edinburgh, EH9 3HJ, UK

22 University of Padova, Department of Astronomy, Vicolo Osservatorio 3, 35122 Padova, Italy

23 Leiden Observatory, Leiden University, PO Box 9513, 2300 RA Leiden, The Netherlands

24 Oxford Astrophysics, Denys Wilkinson Building, University of Oxford, Keble Road, Oxford, OX1 3RH, UK 